\title{
ROLE OF MAGNETIC RESONANCE IMAGING IN PAEDIATRIC EPILEPSY
}

\author{
M. Vijaya Kumari' ${ }^{1}$ J. S. Aswini Jyothi' ${ }^{2}$ A. Suman Chandra ${ }^{3}$, V. Pushpanjali ${ }^{4}$, N. Jayalatha ${ }^{5}$ \\ ${ }^{1}$ Associate Professor of Radiology, Osmania Medical College/Hospital, Hyderabad. \\ ${ }^{2}$ Assistant Professor of Radiology, Osmania Medical College/Hospital, Hyderabad. \\ 3 Professor of Radiology, Osmania Medical College/Hospital, Hyderabad. \\ ${ }^{4}$ Postgraduate Student of Radiology, Osmania Medical College/Hospital, Hyderabad. \\ 5 Professor of Radiology, MNJ Cancer Hospital, Osmania Medical College/Hospital, Hyderabad.
}

\section{ABSTRACT}

\section{BACKGROUND}

Aims and Objectives

1. To identify structural abnormalities in the brain associated with Epilepsy.

2. To study the spectrum of MRI findings in patients with Epilepsy.

3. To study the aetiological factors of Epilepsy.

4. To evaluate the diagnostic efficacy of a standard MRI of the brain in children with epilepsy.

\section{MATERIALS AND METHODS}

We prospectively evaluated 73 patients with clinical impression of epilepsy and who had undergone MRI imaging of the brain from Oct 2012 to 2014 and they were included in the study after an informed consent. The study group comprised of 45 males and 28 females in the age group ranging from 0 to 16 yrs. The study was conducted in the Department of Radiology, Osmania General Hospital, Hyderabad, Telangana. Imaging was done with 1.5 Tesla electromagnet (General Electrical Medical Systems).

\section{RESULTS}

MRI has become indispensable in the diagnostic work-up of epileptic patients, who present with recurrent seizures which is amenable to both medical and surgical treatment. About $15 \%-30 \%$ of patients with partial seizures are refractory to pharmacotherapy, which mandates the need to evaluate these patients with imaging studies to identify possible structural abnormalities, which may be responsible for seizures. Identification of a structural substrate on magnetic resonance imaging guides further management as the chance of being considered for surgical treatment is greatly enhanced when a structural abnormality is found on magnetic resonance imaging. ${ }^{26}$ Approximately, $60 \%$ of patients become seizure free after surgery, the seizure free surgical outcome being $67 \%$ for mesial temporal sclerosis, $75 \%$ for neoplasms and $58 \%$ for cortical dysplasia. ${ }^{27}$

\section{CONCLUSION}

The present study was performed in seventy three patients in the paediatric age group from 0 - 16 years who presented with clinical presentation of seizures, either partial or generalised. They were imaged using an MRI epilepsy protocol. Magnetic resonance imaging should be considered in the initial evaluation of patients presenting with seizure, particularly intractable focal seizures because of its high sensitivity for epileptogenic substrates, superior soft tissue contrast, multiplanar capability, lack of beam hardening artefacts, lack of ionising radiation.

\section{KEYWORDS}

MRI, Epilepsy, Phakomatosis, Neurocysticercosis, Tuberculosis, Gliosis, Arteriovenous Malformations, Leukodystrophy, Cerebral Haemangiomas.

HOW TO CITE THIS ARTICLE: Kumari MV, Jyothi JSA, Chandra AS, et al. Role of magnetic resonance imaging in paediatric epilepsy. J. Evolution Med. Dent. Sci. 2017;6(6):494-509, DOI: 10.14260/jemds/2017/107

\section{BACKGROUND \\ Epilepsy is a chronic condition characterised by recurrent seizures unprovoked by an acute systemic or neurological insult. An epileptic seizure is a clinical manifestation of an abnormal, excessive neuronal activity arising in the grey matter of cerebral cortex.}

Financial or Other, Competing Interest: None.

Submission 20-07-2016, Peer Review 07-08-2016,

Acceptance 12-08-2016, Published 19-01-2017.

Corresponding Author:

Dr. J. S. Aswini Jyothi,

Flat: 204, Vasavi Bhuvana Apartments,

H: 8-3-981/1,3,4,6,8,10,11

Srinagar Colony,

Hyderabad-500037,

Telangana.

E-mail: jyothijs@yahoo.com

DOI: $10.14260 /$ jemds $/ 2017 / 107$

(c) (i) $\ominus$
The incidence of epilepsy is approximately 0.3 to $0.5 \%$ and prevalence of epilepsy is estimated at 5 to 10 persons per 1000. It is age dependent and higher in children than young adults.

The main causes of epilepsy in children are cortical malformations (hemimegalencephaly, cortical dysplasia, lissencephaly, etc.) and phakomatoses (Tuberous sclerosis, Sturge-Weber disease, neurofibromatosis type 1, etc.), perinatal ischaemia, infections, mesial temporal sclerosis, metabolic diseases and tumours.

Imaging indications are precise, which includes partial seizures and an abnormal electroencephalogram. Twenty-five percent of these epilepsy cases are pharmaco-resistant. Indeed, MRI is essential to consider surgical treatment allowing neurosurgeon to localise potential epileptogenic anatomic lesions. In order to diagnose and find the aetiology of the lesion, there are many neuro-radiological investigations that can be utilised. These include pneumoencephalography, 
CSF examination, carotid angiography, EEG, CT and MRI.

Although, the treatment of an isolated seizure is directed towards the immediate underlying metabolic or neurologic derangement, epilepsy usually requires long-term pharmacotherapy or in selected cases neurosurgical intervention to eliminate or reduce recurrent seizures.

Magnetic Resonance Imaging (MRI) is the current imaging tool of choice in the investigation of patients with seizures. The advent of high resolution MRI with a dedicated epilepsy protocol has significantly increased the chances of identifying a cause resulting in a positive clinical impact on the management of these patients. MRI identifies structural defects that require urgent treatment such as high-grade gliomas and arteriovenous malformations, subtle structural abnormalities such as Hippocampal sclerosis and malformations of cortical development. Identification of these conditions has long-term therapeutic and prognostic implications with regard to treatment options and the possibilities of remission or intractability.

\section{MATERIALS AND METHODS}

We prospectively evaluated 73 patients with clinical impression of epilepsy and who had undergone MRI imaging of the brain from Oct 2012 to 2014 and they were included in the study after an informed consent. The study group comprised of 45 males and 28 females in the age group ranging from 0 to 16 yrs. The study was conducted in the Department of Radiology, Osmania General Hospital, Hyderabad, Telangana.

Imaging was done with 1.5 Tesla electromagnet (General Electrical Medical Systems).

\section{Inclusion Criteria}

73 patients of paediatric age group with reference for seizures were subjected to magnetic resonance imaging at Osmania General Hospital, Hyderabad, between 2012 and 2014 and were included in the study.

\section{Exclusion Criteria}

Patients with following features were excluded from the study.

\section{General}

1. Cardiac pacemaker.

2. Cerebral aneurysmal clips.

3. Metal implants.

4. Hearing aids.

\section{Specific}

1. Febrile seizures.

2. Seizures due to dehydration.

\section{METHOD OF DATA COLLECTION}

\section{Criteria for Patient's Selection}

The patients selected for the study were clinically diagnosed cases of seizures as per the criteria laid down by the ILAE 1981.

A detailed history was taken and clinical examination was done. The points noted were duration of illness, type of seizures and any associated illness. Detailed clinical and neurological examination was done to find any neurological deficit. Based on the history and examination, a clinicoaetiological diagnosis was made. Biochemical investigations were done as per the proforma and were found to be within normal limits. All the patients underwent MRI scanning. The procedure was briefly explained to the patient or attendant including the risks of contrast examination.

\section{Methods}

Clinical history of each patient was recorded. A detailed proforma was filled up and all the patients underwent investigations as under.

\section{Routine Investigations}

- Hb, TLC, DLC, ESR, Urine routine examination

- Blood urea, Sugar, Serum creatinine, Serum calcium

- Liver function tests

- Fundus examination

- X-ray chest - PA view

\section{Special Investigations}

- MRI scan of brain (Plain/contrast)

\section{Materials}

- Whole body MRI scanner

- Contrast media gadolinium (Gadobenate Dimeglumine)

- Emergency drugs like Inj. Avil, Dexamethasone and Adrenaline, etc.

- Syringes $5 \mathrm{~mL}, 10 \mathrm{~mL}$ and $20 \mathrm{~mL}$.

\section{Equipment}

1.5 Tesla General Electrical Medical Systems.

\section{Technique of Examination}

All patients screened before entry into the MRI scanning room for ferromagnetic objects, cardiac pacemakers and aneurysmal clips, etc.

Patients were examined in the supine position on the MRI machine after proper positioning and immobilisation of the head was obtained. The paediatric head coil was used for the scan. Initial topogram of the head was obtained and sequences were planned according to the MRI epilepsy protocol.

MRI protocol at $1.5 \mathrm{~T}$ includes the entire brain from nasion to inion, conventional routine $5 \mathrm{~mm}$ slice thickness, T1W and $\mathrm{T} 2 \mathrm{~W}$ axial sequences using spin echo and gradient echo techniques were obtained with TR of $5.51 \mathrm{~ms}$ and TE of 1.69 $\mathrm{ms}$ and the interslice gap was $5.2 \mathrm{~mm}$ for most of the cases and $1 \mathrm{~mm}$ for few cases. Coronal oblique T1 weighted SPGR images were obtained. Protocol also includes coronal and axial FLAIR sequences with $5 \mathrm{~mm}$ slice thickness and $1 \mathrm{~mm}$ interslice gap. A conventional thin slice, T2 weighted axial and coronal sequence was also obtained. Paramagnetic contrast agent, gadobenate dimeglumine was used if a known tumour or vascular malformation identified and also used in neurocutaneous syndromes. Dosage used as $0.1 \mathrm{mg} / \mathrm{kg}$ wt.

As a precautionary measure, resuscitation apparatus and emergency drugs were kept ready. The scans were studied in detail on monitor and finally films were taken for permanent record. MRI findings were recorded in all patients as per the proforma. Every effort was made to make sure of high quality scans and to avoid artefacts.

MR imaging has emerged as the more diagnostically valuable tool because of its excellent soft tissue contrast, allowing for detailed depiction of anatomy, freedom from beam - hardening artefact in basal brain that occur with CT and capacity for multiplanar imaging. 


\section{Aetiology}

The causes of epilepsy can be separated into genetic and acquired disorders. Some genetic causes can result in structural changes that are readily identifiable by crosssectional imaging. There is also clearly a group of patients that require imaging, such as those with a neurocutaneous syndrome or those with infantile spasms where there is a high likelihood of an underlying brain abnormality. The causes of acquired epilepsy are numerous and include all broad groups of abnormality such as vascular, neoplastic, degenerative, traumatic, post-infective and metabolic causes. MRI is generally performed to define the pathological process, be it structural or owing to changes in the local chemical environment such as oedema or gliosis.

Structural neuro-imaging plays an important role in the evaluation, management and treatment of the child with epilepsy. The role of neuro-imaging is to detect an underlying cerebral lesion that may be casually related to the child seizure disorder. MRI is the elective of all structural imaging tools and is considered superior to CT in terms of its sensitivity and specificity of identifying subtle abnormalities.

The principal role of MRI in the definition of the structural abnormalities that underlie seizure disorder (tumours, malformations of cortical development, hippocampal sclerosis, neurocutaneous syndromes, vascular malformations, traumatic lesions, strokes) and to contribute to classification of different epilepsies and epileptic syndromes in order to provide an accurate prognosis for patients.

As per the International League Against Epilepsy Guidelines for Neuroimaging in the Epilepsy patients (1977), which recommends a dedicated epilepsy protocol, MRI for all patients with new-onset seizure or newly diagnosed epilepsy in the non-emergent setting was done.

Epilepsy protocol, which consists of the following sequences:

- T2 Axial Fast Spin Echo (FSE).

- Fluid Attenuated Inversion Recovery Sequence (FLAIR) Axial.

- Diffusion Weighted Sequence (DWI).

- T1 Inversion Recovery (IR) Sequence - Axial.

- T1 Inversion Recovery (IR) Coronal Oblique - 3 MM Slices.

- T2 Coronal Oblique - 3 MM Slices.

- T1 Spoiled Gradient Recalled Echo - Sagittal.

- (3D Magnetisation Prepared Rapid Gradient Echo (MPRAGE).

- Gadolinium was used when a focal lesion/tumour was detected.

\section{IMAGING SPECTRUM OF EPILEPSY NEUROCYSTICERCOSIS}

Neurocysticercosis (NCC) is a clinical condition characterised by the parasitic involvement of the Central Nervous System (CNS) by the larval stage of the pork tapeworm Taenia solium. Humans become the intermediate and definitive host in the life cycle of the tapeworm by ingesting its eggs from contaminated water or uncooked food.

Neurocysticercosis results from infestation with larval form of pig tapeworm, Taenia solium. Neurocysticercosis can be seen in parenchyma, ventricles, subarachnoid spaces or as combination of these. Seizures are the result of the inflammatory response to the dying and degenerating parasite.
Clinical findings depend on number, size, localisation, viability and stage of cyst degeneration.

\section{MR Imaging Features}

MR imaging protocol included the following sequence in all patients: axial spin-echo (SE) T1-weighted imaging, fast spinecho (FSE) T2-weighted imaging, Axial FLAIR imaging and axial/sagittal/coronal SE T1-weighted imaging after injection of contrast, MRS and DWI.

\section{MR Imaging Analysis}

MR imaging findings are depending on the stage of evolution of the larva and on the fact that a single patient can present lesions in different stages of the disease simultaneously. Four recognised stages are the following.

\section{The Vesicular Stage}

The cysticercus is viable and elicits few inflammatory changes in the surrounding tissues. In this stage, the cyst has a thin wall and the cyst fluid has signal intensity similar to that of CSF in all sequences. In viable cysts, an eccentrically located mural nodule represents the scolex. No contrast enhancement or oedema seen.

FLAIR images detect a significantly higher number of scolices than other sequence, which is helpful for diagnosis of NCC. FLAIR image shows the scolex as an eccentrically punctuate bright signal intensity within the cysts.

Revised diagnostic criteria for NCC consider that cystic lesions showing the scolex as an absolute criterion for diagnosis of the disease.

In the colloidal stage as an inflammatory reaction develops around the cysticercus its wall thickness and the vesicular fluid takes on a gelatinous colloidal aspect. Cystic fluid now has a signal intensity slightly distinct from that of CSF in all sequences. The scolex begins to show signs of degeneration and a gradual decrease in the size of the lesion is noticed. Contrast enhancement usually peripheral and perilesional oedema are seen. Slightly hyperintense on T1WI, T2WI and FLAIR images.

Granular nodular stage, the parasite is already dead and retracted, the lesion begins to mineralise. It becomes semisolid, as it is progressively replaced by granulomatous tissue. There is nodular or ring enhancement of the lesion and oedema is less extensive.

In the nodular calcified stage, the lesion is completely mineralised. These lesions appear as small nodules with hypointensity on T2-weighted and $\mathrm{T} 2 *$ - weighted images and better detected on CT studies. Some calcified lesions present persistent contrast enhancement on MR imaging, others may lead to active inflammation during therapy.

Besides parenchymal NCC the second most common site is ventricular, especially in the fourth ventricle. The signal intensity of the cyst is slightly different from that of CSF, usually without enhancement. Hydrocephalus is commonly detected and can be related to the clinical presentation of ventricular NCC due to obstruction of CSF flow. MR imaging shows slight hyperintensity on T1WI, better demonstrate intraventricular cyst and scolex. Subarachnoid NCC usually involves the basal cisterns and sylvian fissures.

These cysts have a signal intensity similar to that of CSF and usually do not enhance after the use of gadolinium. There is associated mass effect causing local subarachnoid 
enlargement and a multiloculated appearance. The scolex is rarely seen.

MRS has been described in cases of large cysticercosis cyst, which shows decreased NAA peak and increased lactate, succinate, acetate and alanine levels.

L. T. Lucato et al studied MR images from 115 patients during 3-year interval and compared the potential for detection of NCC lesions and specifically of the scolex.

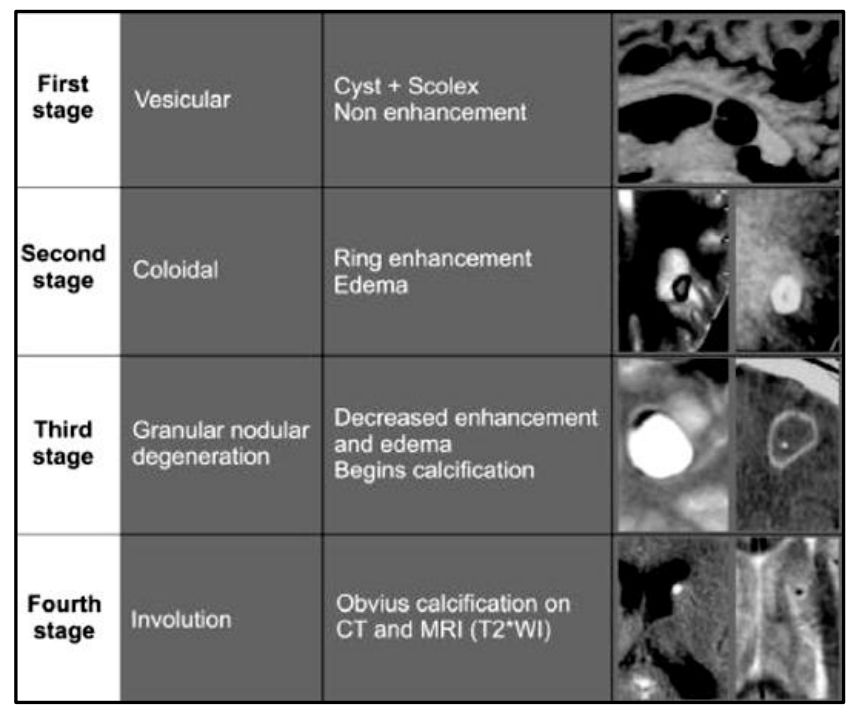

Stages of Cysticerci on MRI

\section{TUBERCULOMAS}

Although, the overall incidence of tuberculosis of the central nervous system has declined, it remains an important disease process in many parts of the world. Tuberculosis may affect any part of the nervous system acutely or chronically and focally or diffusely. Tuberculous meningitis and tuberculoma are the two most important manifestations of tuberculosis of the central nervous system.

Tuberculosis is a chronic granulomatous infection caused by mycobacterium. It is characterised by caseous central necrosis and presence of multinucleated giant cells in the granuloma. CNS tuberculosis can involve the meninges and brain parenchyma, the leptomeningeal version can cause hydrocephalus, neuropathies and deep gray matter infarction. Parenchymal tuberculomas can be single or multiple. The MR imaging findings are nonspecific and depend on the host hypersensitivity reaction to the bacillus. The MR features depend upon whether the granuloma is non-caseating with a solid centre or caseating with liquid centre.

\section{MR Features}

The non-caseating granuloma is usually iso/hypointense on $\mathrm{T} 1 \mathrm{~W}$ and hyperintense on $\mathrm{T} 2 \mathrm{~W}$ images. These granulomas show homogeneous enhancement after injection of contrast agent. The caseating solid granulomas appear relatively isointense/hypointense on T1W images with isointense/hyperintense rim and isointense to hypointense on $\mathrm{T} 2 \mathrm{~W}$ images. These lesions show rim enhancement on postcontrast T1W imaging. MR spectroscopy has been found to be specific for intracranial tuberculomas when combined with imaging. Intracranial tuberculomas are characterised by a spectral pattern that primarily involves long chain lipids with a 0.9 to $1.6 \mathrm{ppm}$ peak range associated with a virtual absence of all brain metabolites, which are normally present.
The commonest aetiology of new onset partial or generalised convulsive seizures is NCC occurring in clusters followed by granuloma. Majority of the epilepsy are generalised type with three major risk factors being brain anoxia, brain infection and traumatic brain injury. Generalised seizures are of two types, convulsive and non-convulsive. The common convulsive type is the tonic-clonic, less common is a purely tonic, purely clonic or clonic-tonic-clonic seizures.

Gulati et al ${ }^{1} 1991$ studied 170 children with chronic epilepsy in which MRI revealed 64 tuberculomas, 27 cases of cysticercosis and 3 gliomas.

H. S. Das et al studied 21 cases with intracranial tuberculoma (MR studies) showed 22 low signal intensity on T2WI, 5 had lesions with central high signal representing caseating granuloma.

Cicek Bayindir et al in a retrospective study of 23 cases of CNS tuberculoma showed that contrast enhanced MRI had an opportunity to diagnose the localised lesions, meningeal enhancement and brain stem enhancement.

\section{VASCULAR LESIONS: CEREBRAL INFARCTION}

Cerebrovascular disease is one of the common cause of epilepsy and stroke being the common cause of seizures. Bladin and Colleagues showed that incidence of seizures varies with underlying pathology and about one-third of people with post stroke seizures develop recurrent seizures.

Intracerebral haemorrhage associated with highest incidence of post stroke seizures and transient ischaemic attack is associated with lowest incidence (3\%).

Seizures after haemorrhagic strokes due to irritation caused by products of blood metabolism. Late onset seizures are associated with persistent changes in neuronal excitability and gliotic scarring.

In ischaemic strokes involving multiple sites, cortical damage and hippocampus involvement are factors that predict the likelihood of developing post stroke seizures.

Gliosis: Gliosis is an astrocytic response to tissue damage and is the end result of various focal or diffuse CNS injuries trauma, infection, infarctions which can be focal/diffuse.

Acute Infarction: Characterised by,

- Lesion in the arterial distribution.

- High intensity in FLAIR/T2WI.

- Gyri Swollen, Sulci effaced.

- Absence of arterial flow void.

- Subcortical white matter hypointensity.

- Intravascular contrast enhancement.

The increase in vasogenic oedema results in brain swelling and well appreciated in cortical regions as gyri swelling/sulci effacement. GE imaging is highly sensitive to susceptibility variations that accompany the intraparenchymal haemorrhage.

Chronic Infarction is characterised by increased signal intensity with atrophic changes seen as hyperintense signal on T2WI/FLAIR.

DWI and perfusion WI techniques help in assessing hyperacute ischaemic changes.

DWI and ADC image read together, hyperintensity on DWI and hypointense signal on ADC of the corresponding area suggestive of acute infarction. 


\section{Vascular Malformations}

Commonly present in the 2nd and 3rd decade. Presenting symptoms include headache, seizures, focal ischaemic neurological deficits or haemorrhage. Vascular malformations constitute $5 \%$ of epilepsy patients.

\section{Arteriovenous Malformations (AVM)}

AVMs are congenital, developmental anomalies of blood vessels. AVMs consist of a tangle of blood vessels lacking intervening capillary network and leading to direct arteriovenous shunting of blood.

The possible mechanism for seizure generation include focal cerebral ischaemia from steal phenomena due to arteriovenous shunting.

Gliosis and hemosiderin deposition from subclinical haemorrhage in the brain parenchyma.

\section{MR Features}

T1- and T2-weighted images demonstrate serpiginous flow voids with areas of $\mathrm{T} 2$ prolongation in the adjoining brain parenchyma.

\section{Cavernous Malformations}

Cavernous malformations are composed of wellcircumscribed vascular spaces containing blood in various stages of evolution. The absence of any intervening neural tissue within the lesion is the hallmark of this lesion.

\section{MR Features}

The typical MR appearance of a cavernous malformation is popcorn-like with a heterogeneous hyperintense signal centrally, on all pulse sequences, surrounded by a rim of low signal intensity from hemosiderin. Because hemosiderin results in magnetic susceptibility artefacts, sequences that are more affected by magnetic susceptibility artefacts will tend to have the greatest sensitivity for detecting small cavernomas. Thus, gradient echo images have a much higher sensitivity when compared with conventional or fast spin echo sequences.

Typical MR imaging shows popcorn lesion with a hemosiderin ring. The lesions are almost completely black on the gradient echo due to blooming artifacts. T2* and susceptibility weighted imaging markedly increase the sensitivity of MRI to detect small cavernomas. Cavernomas are associated with developmental venous anomalies seen as curvilinear flow void on coronal T2WI.

Requena et al (1991) studied cavernomas of central nervous system and described that cavernomas have the characteristic appearance of a range of blood products. The central part contains areas of high signal on T1 and T2WI reflecting oxidised haemoglobin with darker areas on T1weighted images due to deoxyhaemoglobin. The ring of surrounding hemosiderin appears dark on a T2-weighted image. There may be calcification, which usually appears dark on T1- and T2-weighted images.

\section{DEVELOPMENTAL DISORDERS}

\section{Mesial Temporal Sclerosis (MTS)}

Hippocampus is an arched structure found on the medial surface of the temporal lobe. Hippocampal sclerosis is the most common entity associated with medically intractable temporal lobe epilepsy. Regional neuronal loss and gliosis lead to reorganisation of neuronal circuit within the hippocampus, which consequently results in an epileptogenic focus.

This is characterised by marked hippocampal neuronal depletion, loss of inhibitory hippocampal interneurons, synaptic reorganisation.

\section{MR Features}

The coronal T2-weighted images and FLAIR are most sensitive for detecting mesial temporal sclerosis. FLAIR provides images with $\mathrm{T} 2$-weighted contrast and complete suppression of high signal intensity of CSF. Incorporation of a FLAIR sequence into the routine MR evaluation of patients with epilepsy is recommended.

\section{Primary Signs}

- A small atrophic unilateral hippocampus.

- Hyperintensity on both T2W and FLAIR images.

- Loss of hippocampal internal architecture and that of normal digitations of the head.

\section{Secondary Signs}

- Unilateral atrophy of the mammillary body, fornix columns (circuit of papez) and the amygdala.

- Increased T2W signal in the anterior temporal lobe white matter with loss of grey-white demarcation in the ipsilateral anterior temporal lobe.

- Unilateral dilatation of the temporal horn (a less reliable secondary sign).

- Unilateral atrophy of the collateral white matter bundle.

\section{Volumetric MR Imaging}

Quantitative evaluation of hippocampal volume has been found to marginally increase the sensitivity over visual analysis in detection of hippocampal sclerosis.

Measuring size can be accomplished by manually tracing the hippocampus. The normal, ipsilateral hippocampal volume is approximately $2.8 \mathrm{MI}$.

\section{T2 Relaxometry}

It is used to quantify the T2 signal in the hippocampus. It measures the decay in signal intensity at different TEs in a series of $\mathrm{T} 2 \mathrm{~W}$ images acquired in the same slice.

\section{MR Spectroscopy}

NAA is a marker of metabolically active neurons and decreased NAA/creatinine or decreased NAA/creatinine + choline ratios, signify neuronal loss and metabolic dysfunction. A decrease in these ratios has been shown to lateralise temporal lobe epilepsy in $65 \%$ to $90 \%$ of patients with bilateral temporal lobe structural abnormalities on MR.

\section{Malformations of Cortical Development}

Malformations of cortical development are commonly identified as causes of epilepsy and neurodevelopmental deficits. MRI demonstrates significantly the malformations in more detail. Neuroimaging Commission of the International League $^{2,3}$ against epilepsy, subdivide malformations as follows:

1. Diffuse cortical malformations: agyria, pachygyria, polymicrogyria, microcephaly, megalencephaly, microdysgenesis. 
2. Focal or multifocal cortical malformations: focal cortical dysplasia, hemimegalencephaly, focal polymicrogyria, tuberous sclerosis.

3. Heterotopias.

Other, more Detailed Classification of MCDs into Four Basic Categories are as follows:

- Abnormal neuronal and glial proliferation.

- Abnormal neuronal migration.

- Abnormal cortical organisation.

- Unclassified miscellaneous group.

Focal cortical dysplasia is among the most common causes of epilepsy attributable to focal cerebral dysgenesis; $60 \%$ FCD is found in temporal lobes.

\section{Lissencephaly}

It is a rare congenital malformation, results from an arrest in normal neuronal migration. It includes a spectrum of malformations from total failure of development of cerebral sulci and gyri to the development of coarse, broad flat gyri with shallow sulci.

Clinically, the patient may present with microcephaly, mental retardation, seizures, feeding problems and hypotonia. MRI demonstrates abnormalities in cortical thickness and distribution, presence of heterotopias, abnormalities of myelination. ${ }^{4}$

Hemimegalencephaly is characterised by abnormal enlargement of a lobe due to marked dysplasia. This condition could be isolated finding or can be associated with neurocutaneous syndromes. Imaging findings include enlargement of affected lobe or hemisphere with increased signal of white matter on $\mathrm{T} 2$ weighted images and usually the lateral ventricle is enlarged in the dysplastic region.

Cortical surface remains smooth and gray matter mantle is broad and contains thickened bands of disorganised neuronal architecture interspersed with zones of cortical necrosis. The interface between white and gray matter is smooth and lacks the normal pattern of interdigitations. ${ }^{5}$

\section{MRI Findings in Patients with FCD include the Following}

- Cortical thickening observed on at least 3 or more contiguous slices.

- Blurring of the gray-white matter junction.

- Increased signal of the underlying white matter on T2WI/FLAIR.

- Often, a linear, curvilinear or funnel-shaped tapering of abnormal signal intensity extending from the cortical white matter junction to the ependymal surface of the lateral ventricle.

- A deep or wide sulcus with thickened gray matter at the depth of a sulcus.

- Broadening of a gyrus.

Polymicrogyria: It is a malformation due to an alteration of the cortical development in the late stage of neuronal migration. The deeper layers of the cortex form multiple small gyri with derangements of normal sulcation. It may be unilateral or bilateral. It is characterised by:

- Numerous small gyri.

- Predilection for sylvian fissure.

- Atrophy, mainly posteriorly.

- Anomalous venous drainage.

\section{MR Features}

It is seen as thickened cortex with poorly developed sulci and irregular margin of the cortical white matter junction, isointense to gray matter on both $\mathrm{T} 1 \mathrm{~W}$ and $\mathrm{T} 2 \mathrm{~W}$ sequences.

\section{NEUROCUTANEOUS SYNDROMES Sturge-Weber Syndrome}

Sturge-Weber syndrome is a sporadic, congenital neurocutaneous syndrome, characterised by the association of ipsilateral facial angioma in the distribution of the trigeminal nerve with angiomatosis of the leptomeninges. ${ }^{6}$ Clinically, patients present with facial angioma, intractable seizures, hemiparesis, hemianopia and mental retardation. A dysgenetic venous system is responsible for most of the imaging and clinical findings in these patients.

Intractable epilepsy is the earliest and most common clinical presentation in these patients. MR can demonstrate the structural abnormalities in the brain, which include:

1. Pial angiomata in the parietal occipital region on postcontrast enhanced images.

2. Cortical calcifications adjacent to the cortex and white matter in the parieto-occipital region, which are depicted as signal voids or hypointense curvilinear structures on GE.

3. Enlarged choroid plexus, especially on post-contrast enhanced images.

4. Atrophy of the ipsilateral cerebral hemisphere (angioma side).

5. Enlarged and elongated globe of the eye.

Schmauser I et al ${ }^{7}$ concluded that MR is the most sensitive test for revealing the full extent of lesions in Sturge Weber syndrome.

\section{Tuberous Sclerosis}

Tuberous sclerosis is an autosomal dominant disorder that results in multiorgan hamartomas.

It is an inherited systemic disease with prominent cutaneous and CNS manifestations with the classic triad of adenoma sebaceum, epilepsy and mental retardation.

The cortical hamartomas are called tubers and are similar to cortical dysplasia. Subependymal nodules are small lesions protruding into the lateral ventricles. Sometimes they are calcified.

\section{MR Imaging Features}

Areas of T1 and T2 signal abnormality that varies depending on the degree of myelination. In neonates, the tubers are seen as regions of subcortical $\mathrm{T} 1$ hyperintensity and $\mathrm{T} 2$ hypointensity, but after about 6 months of age the signal intensity characteristics are reversed.

Ahmet Kemal Firat et al studied 6 tuberous sclerosis cases and identified hamartomas appear iso-hypointense on T1WI, hyperintense in T2 sequences. Diffusion weighted images are used for differentiating hamartomas from normal white matter.

Raymond et al 1995 studied characteristic appearance of tuberous sclerosis in 5 patients with MRI in a series of 100 patients with MCD and epilepsy and identified large tubers commonly show concordance with surface EEG signs in terms of localisation and lateralisation. 


\section{Neoplasms}

Neoplasms are the structural substrate in $3-4 \%$ of patients with epilepsy in the general population. In patients with intractable epilepsy treated with epilepsy surgery, neoplasms account for approximately $20 \%$ of cases.

Neoplasms associated with chronic epilepsy are usually located in the cortex and are not usually associated with mass effect or vasogenic oedema. The temporal lobe is the most common location (68\%).

Neoplasms found in patients with chronic epilepsy include -

- Low-grade astrocytic tumours

- Oligodendroglioma

- Gangliogliomas

- Dysembryoplastic Neuroepithelial Tumour (DNET), and

- A Pleomorphic Xanthoastrocytoma (PXA).

\section{MR Features}

Astrocytomas, fibrillary subtype (WHO Grade 2) are usually illdefined, infiltrative tumours that usually do not enhance with gadolinium.

Pilocytic astrocytomas, on the other hand are well defined, although not encapsulated and a mural nodule is seen after gadolinium enhancement.

Poorly defined increased signal intensity mass on $\mathrm{T} 2$ and FLAIR sequences arising in supratentorial white matter or central gray matter.

Vouri et al studied in a series of 18 patients with seizures and cortical brain lesions on MRI found on MRS that decreasing NAA and an increase in choline were more pronounced in low-grade gliomas. Contrast enhancement is variable.

Oligodendrogliomas are usually peripherally located and may appear cortically based with gyriform calcifications and adjacent changes in the calvaria. They are commonly seen in the frontal or temporal lobe. Gadolinium enhancement is variable.

Heterogeneous intra-axial mass with cortical infiltration and marked cortical thickening.

Gradient echo may show linear/nodular tumoural calcification as foci of hypointensity.

Contrast enhancement has been in about one-half of the cases. Gangliogliomas are most commonly seen in the temporal lobe. They are mixed solid and cystic lesions that are cortically based with minimal or no mass effect. Calcification is often present. Gadolinium enhancement is variable. These lesions can be associated with concomitant cortical dysplasia. On MRI lesions have low signal intensity on T1WI and high signal intensity on T2WI sequences and show enhancement after contrast.

Dysembryoplastic Neuroepithelial Tumours (DNET) are benign, low-grade, multicystic and multinodular cortical based tumours that are seen primarily in children and young adults. A cortically based hypointense nodule can be seen on T1weighted images. Calvarial remodelling may be seen. Cortical dysplasia can be seen in $20 \%-30 \%$ of these tumours. Gadolinium enhancement is variable. If a cortically based, multicystic tumour is seen on MRI, a DNET should be considered.

Pleomorphic Xanthoastrocytoma (PXAs) are superficially located tumours adjacent to the leptomeninges with an enhancing mural nodule. Leptomeningeal involvement is characteristic of this tumour.

\section{MR Features}

Demonstrates superficial solid mass with large cystic component. The solid component usually enhances and is calcified in about half of the cases.

\section{Hypoxic Ischaemic Injury}

Antenatal, perinatal and post-natal vascular events, either hypoxic, ischaemic or haemorrhagic can lead to a wide range of intracranial abnormalities, some of which will be associated with epilepsy. The lesions can be unilateral or bilateral with and without symmetry. Unilateral abnormalities may include porencephaly and focal haemorrhage. Perinatal hypoxicischaemic encephalopathy is a common cause of epilepsy and may result in different features depending on the severity and timing of the injury.

\section{MRI Features}

It shows periventricular leucomalacia, multicystic encephalomalacia, brain stem and basal ganglia changes that represent varying degrees of hypoxic-ischaemic injury. DWI confirms the diffuse restriction.

\section{Cerebral Atrophy}

Huckman et $\mathrm{al}^{9}$ in a study of degenerative brain diseases classified atrophy into 3 types, i.e. cortical, central and combined. This classification was based on the size of the ventricles and cortical sulci.

\begin{tabular}{|c|c|c|}
\hline $\begin{array}{c}\text { Grade of } \\
\text { Atrophy }\end{array}$ & Ventricular Width & Sulci \\
\hline $\begin{array}{c}\text { Borderline } \\
\text { atrophy }\end{array}$ & $16-20 \mathrm{~mm}$ & $<5 \mathrm{~mm}$ \\
\hline Mild atrophy & $16-20 \mathrm{~mm}$ & $6-9 \mathrm{~mm}$ \\
\hline Moderate atrophy & $\begin{array}{c}>20 \mathrm{~mm} \text { or } 16-20 \\
\mathrm{~mm}\end{array}$ & $\begin{array}{c}6-9 \mathrm{~mm} \text { or }>9 \\
\mathrm{~mm}\end{array}$ \\
\hline Severe atrophy & $>20 \mathrm{~mm}$ & $>9 \mathrm{~mm}$ \\
\hline
\end{tabular}

Cortical Atrophy was further Classified as Under

Normal ventricular width was taken to be $<15 \mathrm{~mm}$ and normal cortical sulci width $<5 \mathrm{~mm}$. In the above study, the patients were divided into two categories, i.e. diffuse atrophy and focal atrophy. Diffuse atrophy was further divided into,

1. Cortical: Dilatation of cortical sulci only with normal lateral ventricles.

2. Central: Dilatation of LV only with the normal cortical sulci.

3. Combined: Dilatation of both ventricles and sulci.

\section{MR Features}

The focal and confluent areas of increased signal intensity on conventional T2WI and FLAIR sequences in the white matter and central nuclei.

Selective atrophy of white matter predominates than gray matter in ageing. Diffusion anisotrophy in white matter changes with age; these quantifiable changes may serve as a standard for comparison with neurodegenerative diseases.

S. I. Bekkelund et al studied 32 cerebral atrophy patients and found that general brain atrophy to be present in epileptic patients including those with partial epilepsy. Whether atrophy in epileptic patients occurs as a consequence of disease related factors like hypoxia or treatment with 
antiepileptic drugs has to be investigated in a prospectively designed study.

\section{Cerebral Abscess}

Cerebral abscess results from pathogens growing within the brain parenchyma, initially as a cerebritis and then eventually demarcating into a cerebral abscess. Direct extension of infections was the most common source. More recently haematogenous spread has become most common. Direct introduction by trauma or surgery accounts for only a small minority of causes. Risk factors for haematogenous spread are congenital heart diseases, bacterial endocarditis, lung infection, dental abscess and systemic sepsis.

\section{MRI Features}

MRI is more sensitive and especially with the addition of MRS and DWI far more specific for the diagnosis of cerebral abscesses.

- T1WI central low intensity (hyperintense to CSF)

- Peripheral low intensity (vasogenic oedema)

- Ring enhancement

- Ventriculitis may be present, in which case hydrocephalus will commonly also be seen.

\section{T2/FLAIR WI}

Central high intensity (hypointense to CSF does not attenuate on FLAIR.

Peripheral high intensity (vasogenic oedema).

The abscess capsule may be visible as an intermediate to slightly low signal thin rim.

\section{DWI/ADC}

High DWI signal is usually present centrally.

Often this represents true restricted diffusion (low signal on ADC.

In many instances, high DWI are associated with high ADC signal consistent with $\mathrm{T} 2$ shine through of the central neurotic portion.

\section{MR Spectroscopy}

Elevation of a succinate peak is relatively specific but not present in all abscess cases; High lactate, alanine, valine, leucine and isoleucine levels peak with reduced NAA peak.

\section{Venous Sinus Thrombosis}

The common predisposing factors are puerperium, pregnancy, infection, oral contraceptive, coagulopathies, tumours, trauma, etc. Clinically, most of the patients present with headache, seizures and nausea. Superior sagittal sinus is the most commonly occluded dural sinus followed by transverse, sigmoid and cavernous sinuses. The MR findings vary with clot age. Acute thrombus is isointense with cortex on T1WI, late acute clots are hyperintense on T1WI. Subacute thrombi are typically hyperintense on all pulse sequences.

MR angiography 2D TOF and 2D PC sequences used for screening cerebral venous circulation. Sagittal 2D PC or coronal 2D TOF MRA is best used to evaluate the superior sagittal sinus.

Dural sinus thrombosis is being detected with increasing frequency by MR imaging. The sensitivity of MRI is close to $100 \%$ in detecting AVMs and cavernous malformations. Venous infarcts ${ }^{5}$ are typically subcortical and haemorrhagic.
Retrograde extension of thrombus into cortical or deep medullary veins occur in approximately $40 \%$ cases of dural sinus thrombosis.

\section{MR Findings}

The signal void in the dural sinus or cortical vein is replaced by abnormal signal. The specific appearance varies with the age of the thrombus and sequence parameters. Filling defect following contrast and asymmetric enhancement of nearby vessels seen. MR venogram demonstrates absence of flow related enhancement, which support the diagnosis of sinus thrombus.

\section{Postoperative Imaging}

MR imaging plays a crucial role not only in the presurgical evaluation of patients with medically refractory epilepsy, but also in the postoperative imaging of these patients. MR imaging can determine the extent of surgical resection for epileptogenic substrates and surgical divisions for functional hemispherectomies.

In Patients who have not had Success with Surgery, MR Imaging can Identify

1. Residual substrate at the operative site,

2. Any other previously unrecognised epileptogenic substrates at other locations in the brain, and

3. Persistent connections in functional hemispherectomies.

MR imaging is more sensitive in detecting complications of hemispherectomy, such as extra or intra-axial haemorrhage, hydrocephalus or infections.

Montes J L et al ${ }^{10}$ studied lesionectomy cases with MRI in children presenting with epilepsy and concluded that MRI was better in differentiating neoplastic from developmental lesions. Early consideration of surgery in patients with epilepsy and lesions demonstrated by MRI is suggested.

Van Passechen W. et al ${ }^{11}$ studied 40 patients to evaluate and compare $\mathrm{T} 2$ relaxometry and volumetrics of hippocampus in presurgical evaluation of patient with intractable temporal lobe epilepsy and revealed hippocampal volume asymmetry could be reliably detected on visual inspection of the MRI with hippocampal volume ratio of 0.85 or less. Quantitative MRI reliable for detecting of Hippocampal sclerosis.

P. Salgado et al ${ }^{12}$ studied 6 patients with intracranial tuberculoma and showed MR lesions with prolongation of the $\mathrm{T} 1$ relaxation time in every case. On the T2-weighted sequences, the signal properties of the tuberculoma varied according to the stage of evolution of the lesion. Mature tuberculomas were composed of a dark necrotic center surrounded by an isointense capsule, which was in turn surrounded by oedema. In one patient center of the lesion was hyperintense, probably because of liquefaction and pus formation.

Liu RS et al ${ }^{13}$ studied 120 patients with chronic epilepsy and observed that focal and generalised neocortical atrophy commonly develops in chronic epilepsy and commented that the progressive neuronal loss may be an important factor in understanding epileptogenesis, neurocognitive impairment, Adverse Antiepileptic Drug (AED) effects and psychopathology in patients with seizure disorders.

Phua Hwee Tang et al $^{14}$ studied 22 cerebrovenous thrombosis cases and concluded as abnormal signal replacing 
the hypointense signal void of flowing blood on T1, T2 and FLAIR sequences. The FLAIR shows the filling defect in coronal plane.

The best practice is to obtain MRI in all patients with epilepsy with the exception of those with a definite diagnosis of idiopathic generalised epilepsy or benign rolandic epilepsy of childhood with centro-temporal spikes, the principle role of MRI is in the definition of structural abnormalities that underlie seizure disorders. Hippocampal sclerosis may be reliably identified, quantitative studies are useful for research and in equivocal cases, for clinical purposes. A range of Malformations of Cortical Development (MCD) may be determined. In patients with refractory partial seizures who are candidates for surgical treatment, a relevant abnormality is identifiable using MRI in $85 \%$. It is likely that subtle MCD or gliosis accounts for the majority of the remainder. The proportion of cryptogenic cases will decrease with improvements in MRI hardware, signal acquisition techniques and post-processing methodologies.

Brodtkorb et al15 1992 studied 303 patients with epileptic seizures referred for MRI and found 4.3\% malformations of cortical development.

Lehericy et al ${ }^{16} 1995$ in their study of 222 patients with temporal lobe epilepsy and revealed with MRI comprising FCD, nodular heterotopia, abnormal gyri, hippocampal malformations $(7 \%)$ and concluded that in temporal lobe epilepsy imaging, MRI plays a key role.

Libenson et al studied MRI of brain and spinal cord on a patient with tonic seizure; MRI scan revealed abnormality in the lateral right cerebral peduncle and deep frontal white matter. Serial MRI scan revealed resolution of brain lesions correlating with clinical prognosis.

Petroski $\mathrm{S}$ et al, used MRI for prediction of seizure recurrence in 170 newly treated patients with epilepsy, MRI used as one of the important tool in prospective followup of antiepileptic drug treatment showed lesion associated cases with high risk of seizure recurrence.

Thornton $\mathrm{R}$ et al studied EEG correlated functional MRI was recorded in 76 patients undergoing presurgical evaluation and post-operative outcome in focal epilepsy and the results showed the potential value of EEG and fMRI in presurgical evaluation.

Yao Y Guojun et al conducted MRI study in presurgical evaluation in 23 patients with drug-resistant hypermotor seizures. MRI detected focal abnormalities in six patients.

MRI could get good surgical results after corticoamygdalohippocampectomy.

Toledano $\mathrm{R}$ et al studied MRI and proved mesial frontal lobe lesions in six patients out of ten epilepsy patients. The diagnosis of bilateral akinetic seizures achieved with EEG and other parameters is often supported by locating lesions in brain by MRI.

Sanchetee et al in their study on NCC concluded that MRI is more sensitive in demonstration of non-calcified cerebral lesions.

\section{OBSERVATIONS AND RESULTS}

A total of 73 patients who presented with epilepsy were refered from Niloufer Paediatric Hospital to Radiology Dept. in Osmania General Hospital, and underwent magnetic resonance imaging of the brain in a time period from May 2012 to Sept 2014 were included in the study.

\begin{tabular}{|c|c|c|}
\hline Seizure Type & $\begin{array}{c}\text { No. of } \\
\text { Patients }\end{array}$ & $\begin{array}{c}\text { Percentage } \\
\text { Distribution }\end{array}$ \\
\hline $\begin{array}{c}\text { Partial/Focal } \\
\text { Seizures }\end{array}$ & 43 & $58.9 \%$ \\
\hline $\begin{array}{c}\text { Generalised } \\
\text { Seizures }\end{array}$ & 30 & $41.09 \%$ \\
\hline Total & $\mathbf{7 3}$ & \\
\hline \multicolumn{2}{|c|}{$\begin{array}{r}\text { Table 1: Classification of Cases on the } \\
\text { Basis of Clinical Presentation }\end{array}$} \\
\hline
\end{tabular}

Patients with partial seizures constituted $58.9 \%$ of the study group.

Patients with generalised seizures constituted $41.09 \%$ of the study group.

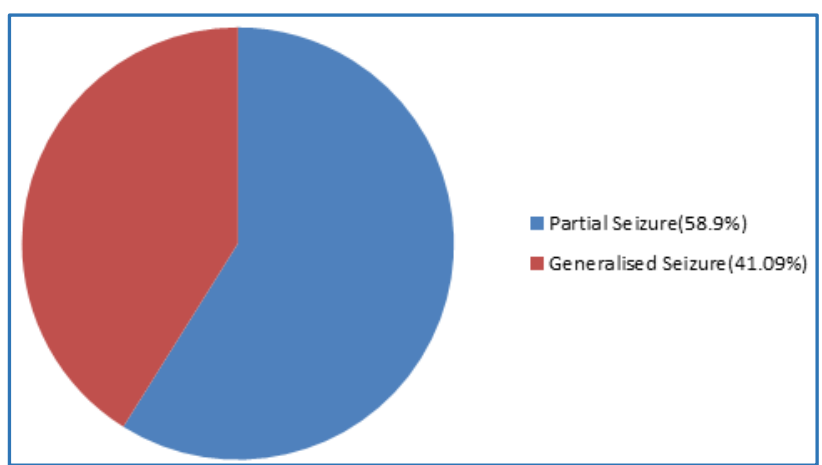

Fig. 1: Classification of Cases on the Basis of Clinical Presentation

\begin{tabular}{|c|c|c|}
\hline Clinical Diagnosis & No. of Patients & Percentage \\
\hline GTCS & 30 & $20.55 \%$ \\
\hline Myoclonic seizures & 2 & $1.37 \%$ \\
\hline Absence seizures & 0 & $0.00 \%$ \\
\hline Simple partial seizures & 18 & $12.33 \%$ \\
\hline $\begin{array}{l}\text { Complex partial } \\
\text { seizures }\end{array}$ & 16 & $10.96 \%$ \\
\hline Temporal lobe seizures & 3 & $2.05 \%$ \\
\hline Febrile seizures & 0 & $0.00 \%$ \\
\hline Neonatal seizures & 0 & $0.00 \%$ \\
\hline Motor seizures & 2 & $1.37 \%$ \\
\hline Tonic seizures & 2 & $1.37 \%$ \\
\hline \multicolumn{3}{|c|}{$\begin{array}{c}\text { Table 2: Distribution of Patients on the Basis of Clinical } \\
\text { Diagnosis of Seizures }\end{array}$} \\
\hline
\end{tabular}

Maximum number of patients presented with partial seizures account to $23 \%$ of the study group. 


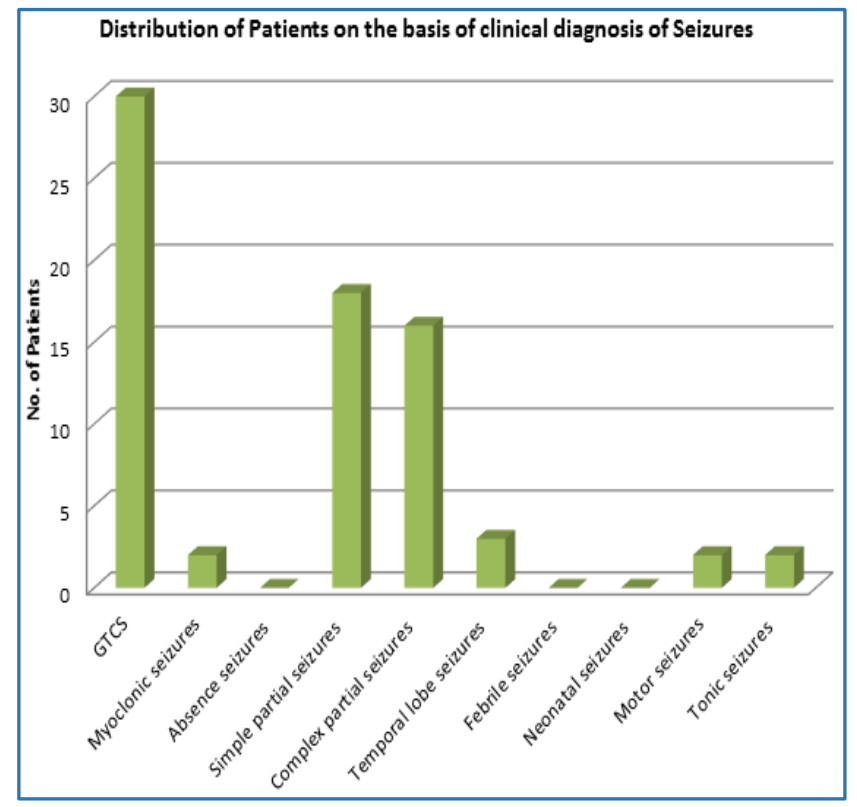

Fig. 2: Distribution of Patients based on Clinical Diagnosis of Seizures

\begin{tabular}{|c|c|c|}
\hline MRI Features & $\begin{array}{c}\text { Partial Seizure } \\
\text { Group }\end{array}$ & $\begin{array}{c}\text { Generalised Seizure } \\
\text { Group }\end{array}$ \\
\hline $\begin{array}{c}\text { MRI with } \\
\text { Findings }\end{array}$ & 31 & 12 \\
\hline Normal MRI & 12 & 18 \\
\hline Total & $\mathbf{4 3}$ & $\mathbf{3 0}$ \\
\hline \multicolumn{3}{|c|}{ Table 3: MRI Findings in Patients with Partial and } \\
Generalised Seizures
\end{tabular}

Among 43 patients with partial seizures, 31 patients had lesions detected on MRI. The diagnostic yield of MRI is $72.09 \%$. The diagnostic yield of MRI in generalised seizure group is $40 \%$.

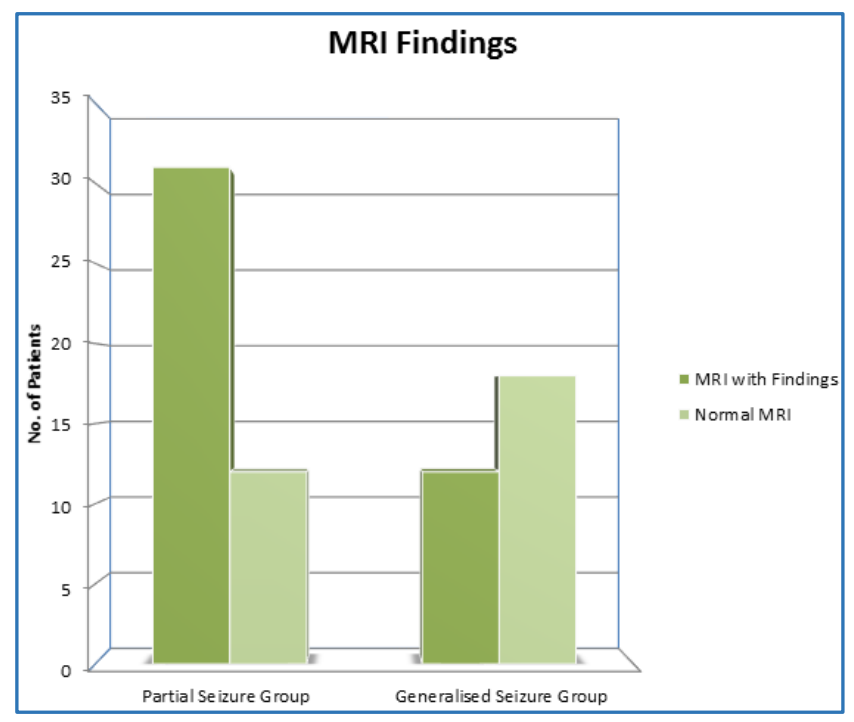

Fig 3: MRI Findings in Patients with Partial and Generalised Seizures

\begin{tabular}{|c|c|c|}
\hline $\begin{array}{l}\text { Epileptogenic } \\
\text { Substrate }\end{array}$ & $\begin{array}{c}\text { No. of } \\
\text { Patients }\end{array}$ & $\begin{array}{c}\text { Percentage of } \\
\text { Distribution }\end{array}$ \\
\hline $\begin{array}{l}\text { Infectious } \\
\text { Aetiology }\end{array}$ & 13 & $30.23 \%$ \\
\hline Gliosis & 6 & $13.95 \%$ \\
\hline Vascular Disease & 5 & $11.63 \%$ \\
\hline Tumours & 4 & $9.30 \%$ \\
\hline $\begin{array}{c}\text { Congenital } \\
\text { Malformations }\end{array}$ & 4 & $9.30 \%$ \\
\hline Phakomatoses & 3 & $6.98 \%$ \\
\hline Encephalitis & 3 & $6.98 \%$ \\
\hline $\begin{array}{l}\text { Mesial Temporal } \\
\text { Sclerosis }\end{array}$ & 2 & $4.65 \%$ \\
\hline Leucodystrophy & 2 & $4.65 \%$ \\
\hline $\begin{array}{c}\text { Vascular } \\
\text { Malformations }\end{array}$ & 1 & $2.33 \%$ \\
\hline Total & 43 & \\
\hline
\end{tabular}

In our study, infections were the most common abnormality identified in $30.23 \%$ of the study group followed by gliosis with $13.9 \%$.

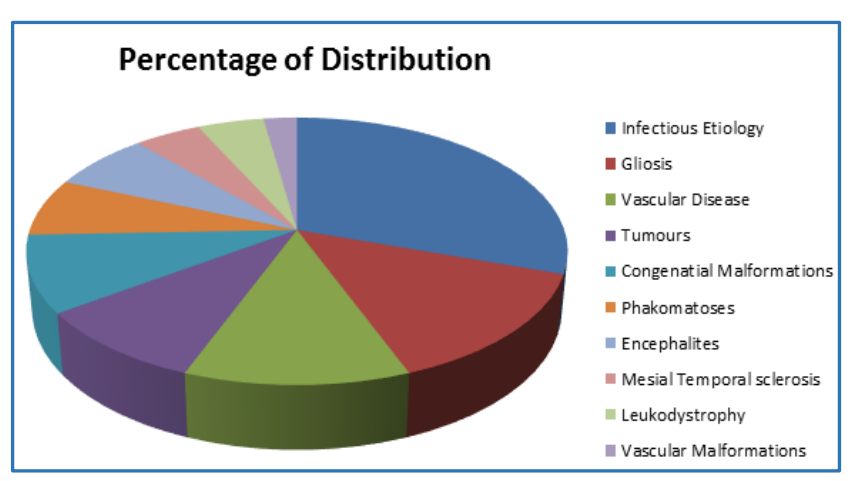

Fig. 4

\begin{tabular}{|c|c|c|}
\hline Sex & No. of Patients & Percentage Distribution \\
\hline Male & 45 & $61.42 \%$ \\
\hline Female & 28 & $38.57 \%$ \\
\hline Total & 73 & \\
\hline \multicolumn{2}{|c|}{ Table 5: Distribution of Cases According to Sex } \\
\hline
\end{tabular}

In our study, the majority were male patients. (61.42\%).

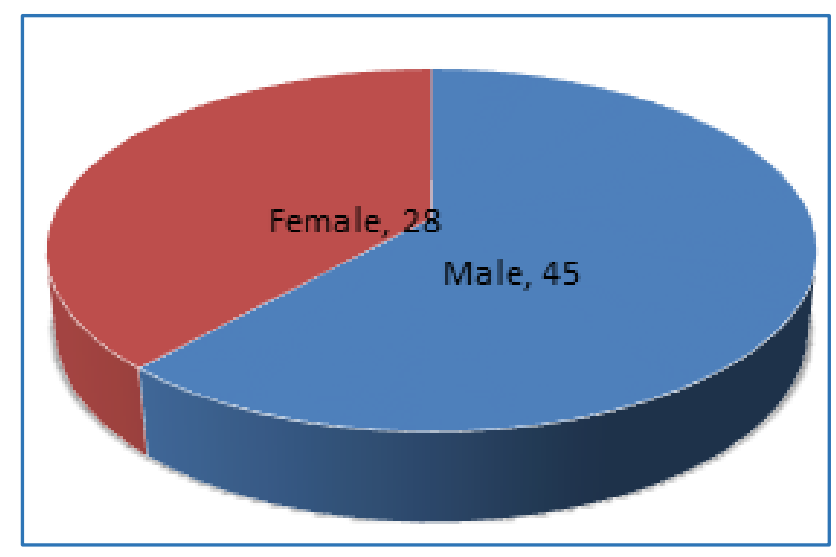

Fig. 5: Distribution of Cases According to Sex 


\begin{tabular}{|c|c|c|}
\hline Age Group & $\begin{array}{c}\text { No. of } \\
\text { Patients }\end{array}$ & $\begin{array}{c}\text { Percentage of } \\
\text { Distribution }\end{array}$ \\
\hline 0 - 1 Years & 14 & $19.18 \%$ \\
\hline 2 - 6 Years & 15 & $20.55 \%$ \\
\hline 7 - 11 Years & 20 & $27.40 \%$ \\
\hline $\begin{array}{c}12-16 \\
\text { Years }\end{array}$ & 24 & $32.88 \%$ \\
\hline Total & $\mathbf{7 3}$ & \\
\hline Table 6: Classification of Cases based on Age Group \\
\hline
\end{tabular}

Majority of the patients belonged to 12 to 16 years' age group in the study, the percentage being $32.88 \%$.

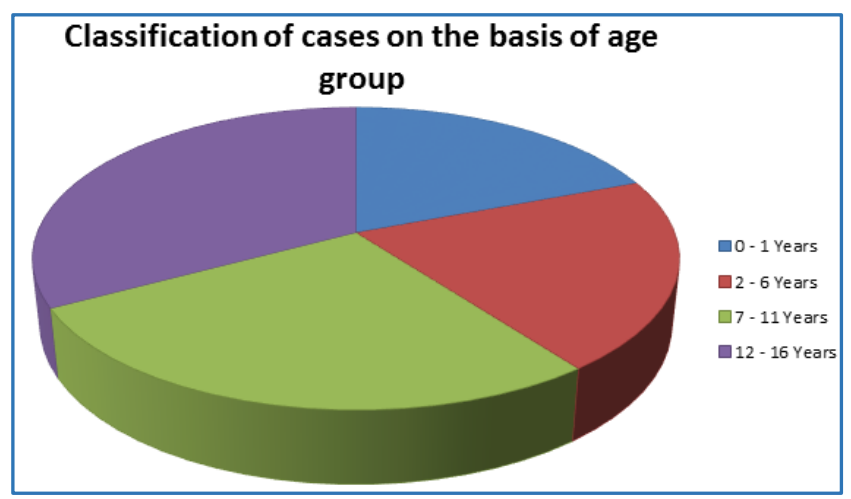

Fig. 6: Classification of Cases on the Basis of Age Group

\begin{tabular}{|c|c|c|}
\hline Duration of Illness & No. of Patients & Percentage \\
\hline$<1$ Month & 6 & $8.22 \%$ \\
\hline 1 - 3 Months & 23 & $31.51 \%$ \\
\hline > 3 Months & 44 & $60.27 \%$ \\
\hline Total & 73 & \\
\hline Table 7: Distribution of Patients based on Duration of \\
Seizures \\
\hline
\end{tabular}

More than half of patients $44 / 73(60.27 \%)$ presented with age over 3 months.

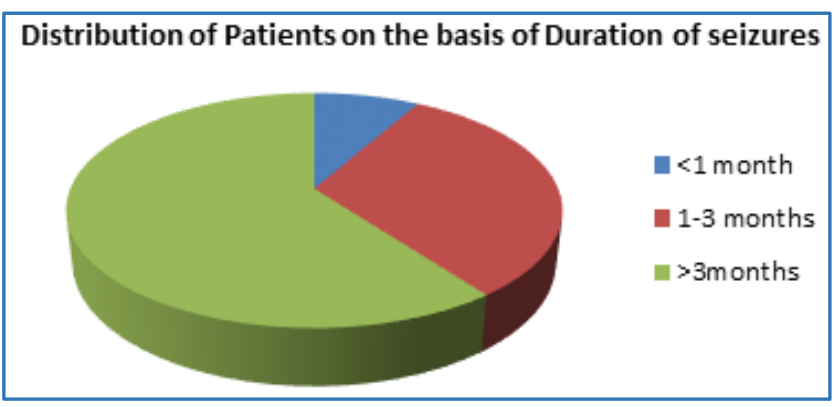

Fig. 7: Distribution of Patients based on Duration of Seizures

\begin{tabular}{|c|c|c|}
\hline $\begin{array}{l}\text { Infectious } \\
\text { Aetiology }\end{array}$ & $\begin{array}{c}\text { No. of } \\
\text { Patients }\end{array}$ & $\begin{array}{l}\text { Percentage of } \\
\text { Distribution }\end{array}$ \\
\hline Tuberculosis & 5 & 38.46 \\
\hline $\begin{array}{l}\text { Neurocysticerco } \\
\text { sis }\end{array}$ & 8 & 61.54 \\
\hline Total & 13 & \\
\hline
\end{tabular}

Neurocysticercosis was seen more frequently than tuberculosis as the infectious aetiology in the study group.

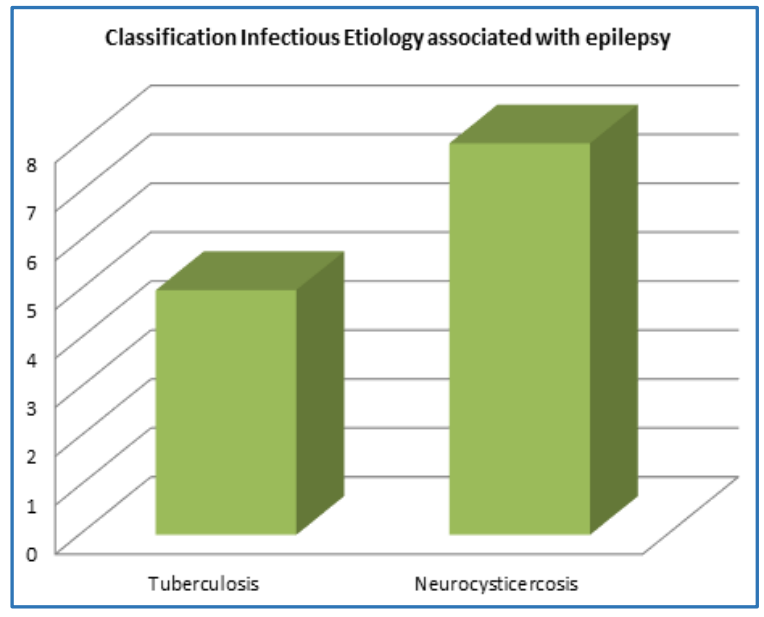

Fig. 8: Classification of Infectious Aetiology Associated with Epilepsy

\begin{tabular}{|c|c|c|}
\hline Aetiology & $\begin{array}{c}\text { No. of } \\
\text { Patients }\end{array}$ & $\begin{array}{c}\text { Percentage of } \\
\text { Distribution }\end{array}$ \\
\hline $\begin{array}{c}\text { Encephalomalacia with } \\
\text { Gliosis }\end{array}$ & 2 & $33.33 \%$ \\
\hline $\begin{array}{c}\text { Periventricular } \\
\text { Leucomalacia }\end{array}$ & 2 & $33.33 \%$ \\
\hline $\begin{array}{c}\text { Hypoxic Ischaemic } \\
\text { Encephalopathy }\end{array}$ & 1 & $16.67 \%$ \\
\hline Porencephaly & 1 & $16.67 \%$ \\
\hline Total & 6 & \\
\hline Table 9: Aetiological Classification of Gliosis Associated \\
with Epilepsy \\
\hline
\end{tabular}

Gliosis is the $2^{\text {nd }}$ most common cause of epilepsy in the study group in which perinatal insult to the brain being the common aetiology followed by periventricular leucomalacia according to $33 \%$.

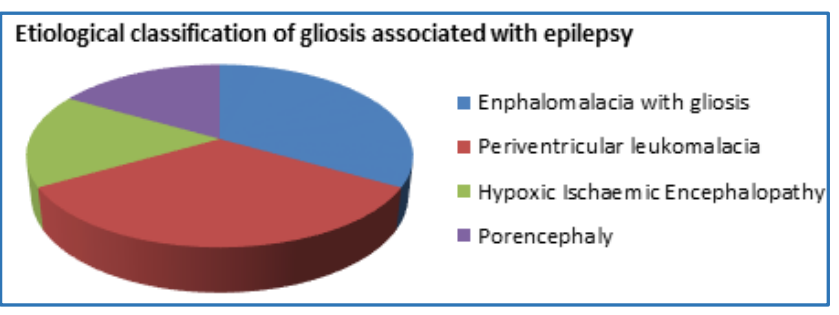

Fig. 9: Aetiological Classification of Gliosis Associated with Epilepsy

\begin{tabular}{|c|c|c|}
\hline Infarct Type & $\begin{array}{c}\text { No. of } \\
\text { Patients }\end{array}$ & $\begin{array}{l}\text { Percentage of } \\
\text { Distribution }\end{array}$ \\
\hline $\begin{array}{l}\text { Venous } \\
\text { Infarct }\end{array}$ & 2 & $40 \%$ \\
\hline $\begin{array}{l}\text { Arterial } \\
\text { Infarct }\end{array}$ & 3 & $60 \%$ \\
\hline Total & 5 & \\
\hline \multicolumn{3}{|c|}{$\begin{array}{c}\text { Table 10: Classification of Vascular Disease Associated } \\
\text { with Epilepsy }\end{array}$} \\
\hline
\end{tabular}




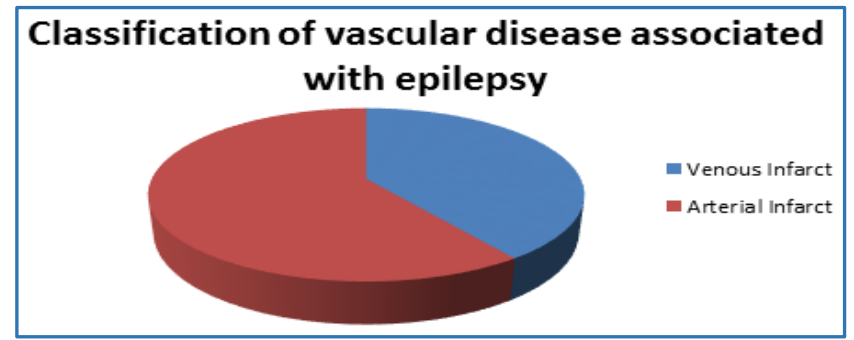

Fig. 10: Classification of Vascular Disease Associated with Epilepsy

\begin{tabular}{|c|c|c|c|c|}
\hline $\begin{array}{l}\text { 꼴 } \\
\text { zo } \\
z\end{array}$ & 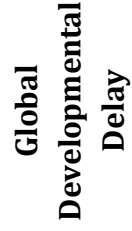 & 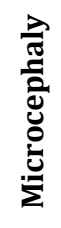 & 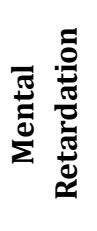 & 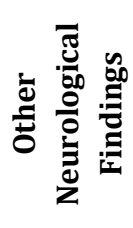 \\
\hline 51 & 6 & 5 & 3 & 8 \\
\hline \multicolumn{5}{|c|}{$\begin{array}{c}\text { Table 11: Various Neurological Findings in Different } \\
\text { Patients }\end{array}$} \\
\hline
\end{tabular}

Neurological examination was normal in 51 patients and only 23 patients showed focal signs.

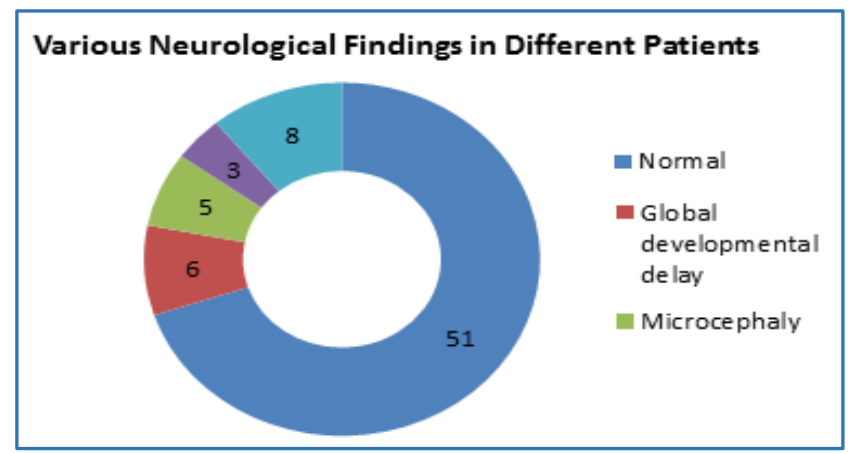

Fig. 11: Various Neurological Findings in Different Patients

\section{CASE 1}

Patient's Name: XXXXX, Age: 2 yrs., Sex: Male

Clinical History: Recurrent seizures and developmental delay. Type of Seizure: Focal seizures. Duration: Since birth. MRI Conclusion: Open-lip schizencephaly with cortical dysplasia.

Final Diagnosis: Developmental malformation - open-lip schizencephaly with cortical dysplasia.

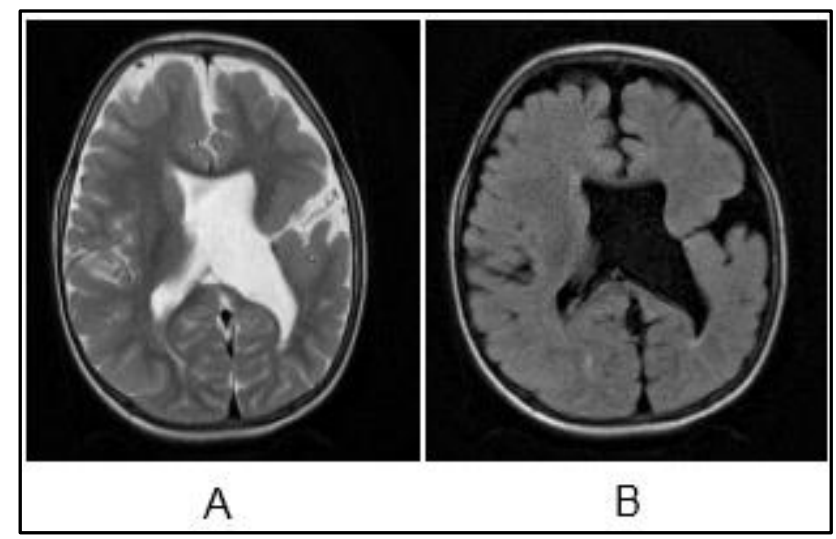

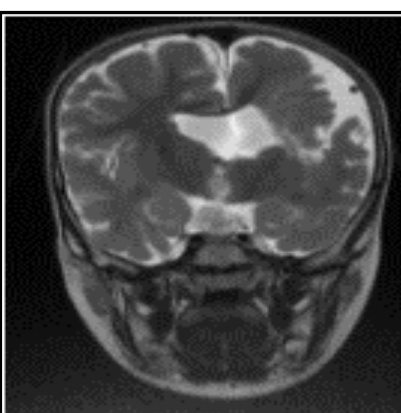

C

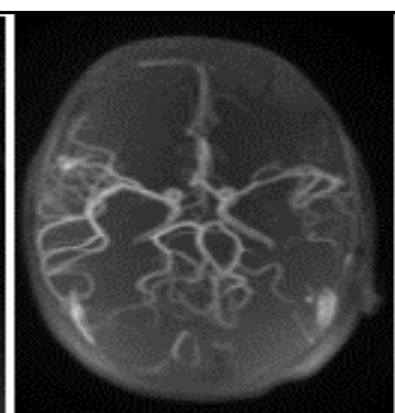

D

A. Axial T2WI and B. Coronal T2W1 Image - Showing decreased volume of left hemisphere and CSF containing cleft lined by grey matter is noted in left parietal lobe communicating with left ventricle, suggestive of open-lip schizencephaly with cortical dysplasia.

C. FLAIR Image - Showing evidence of decreased volume of white matter of left parietal lobe with smooth and thickened gyri of cortex.

D. MR Angiogram - Normal.

CASE 2

Patient's Name: XXXXX, Age: 13 yrs., Sex: Male.

Clinical History: Headache, nausea and recurrent seizures.

Type of Seizure: Intractable partial seizures. Duration: 6 yrs. MRI Conclusion: Space occupying lesion/Tumour in left parietal and temporal lobe.

Final Diagnosis: Dysembryoplastic Neuroepithelial Tumour (DNET) and was proved by histopathology.

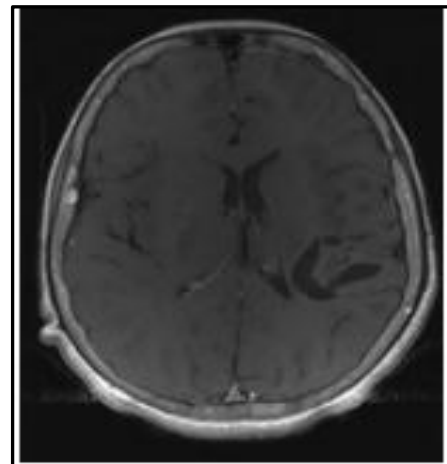

A

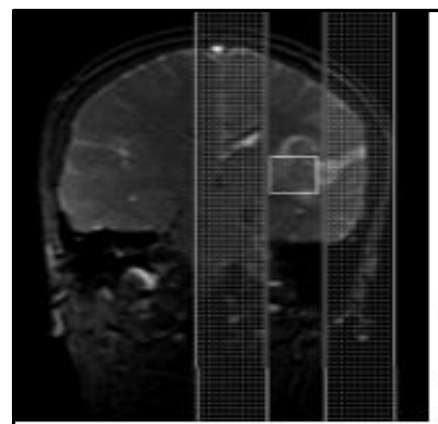

C

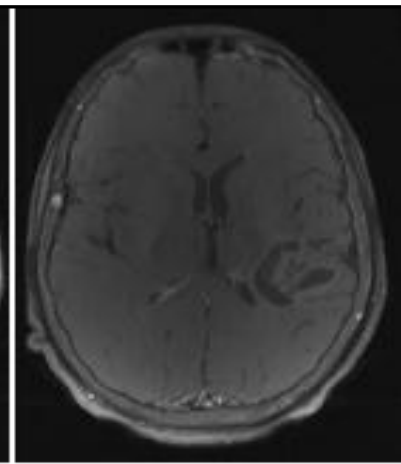

B

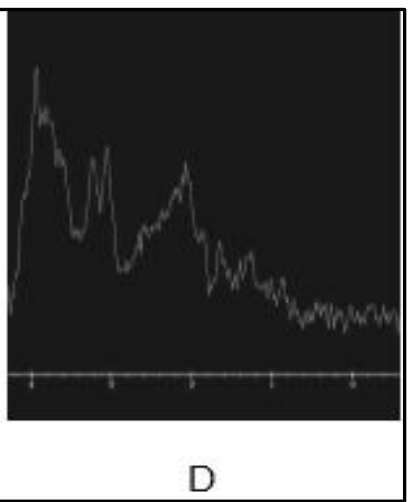


A. Axial T2WI - Showing a heterogeneous altered signal intensity lesion in left temporal and parietal lobe, which is bubbly in appearance with cystic and solid component. The lesion is showing very minimal perilesional oedema and no mass effect.

B. Axial T1W Contrast Image - Showing peripheral and heterogeneous enhancement.

C and D MRS - On long TE sequence showing decrease in NAA and increase in choline-creatinine ratio.

\section{CASE 3}

Patient's Name: XXXXX, Age: 13 yrs., Sex: Male

Clinical History: Fever, headache, vomiting, recurrent seizures.

Type of Seizure: Generalised tonic-clonic seizures.

Duration: 6 months.

MRI Conclusion: Well-defined ASI lesion, which is isointense with hyperintense rim with moderate perilesional oedema noted in left frontal region showing ring enhancement on contrast suggestive of granulomatous disease probably neurocysticercosis.

Final Diagnosis: Neurocysticercosis.

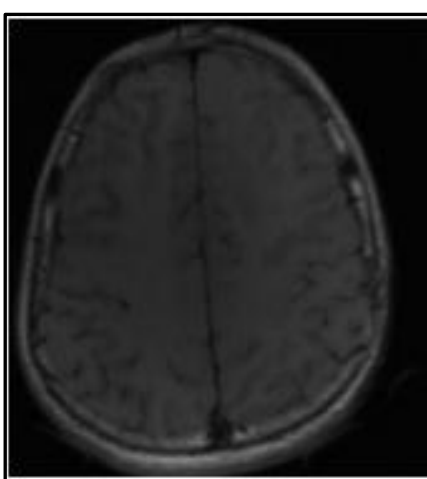

A

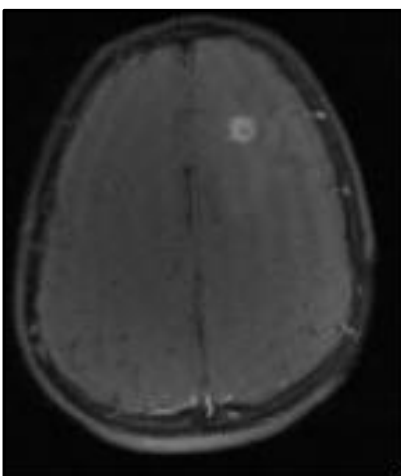

C

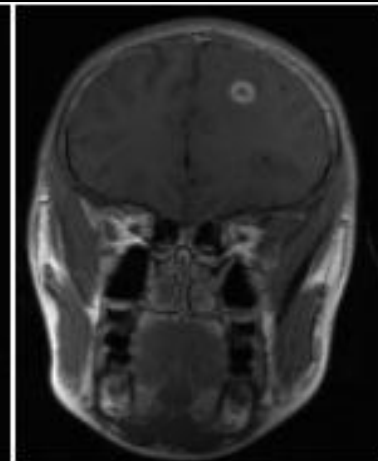

B

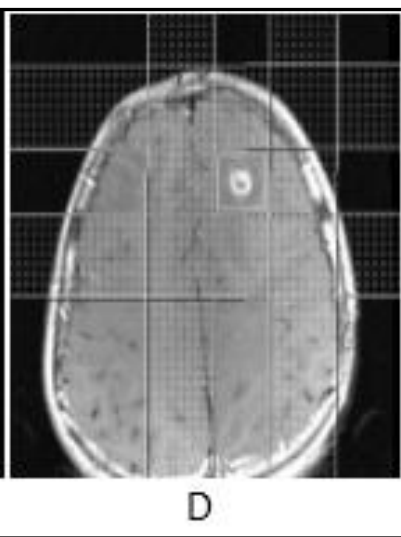

A. Axial T1WI - Showing isointense lesion in left frontal region.

B. Coronal T2WI - Showing well-defined ASI lesion, which is isointense with hyperintense rim with moderate perilesional oedema in left frontal region.

C and D Axial Post-Contrast Image - Showing ring enhancing lesion with a nodule (scolex) within.

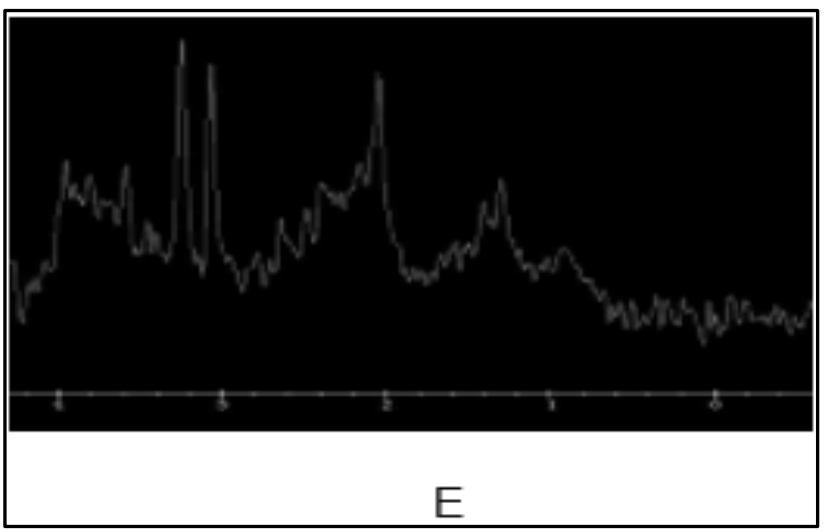

E. MRS - Showing elevated lipid lactate peak.

CASE 4

Patient's Name: XXXXX, Age: 13 yrs., Sex: Female

Clinical History: Recurrent refractory seizures and developmental delay with weakness/hemiparesis of right side of body.

Type of Seizure: Myoclonic seizures.

Duration: Since 1 year of birth

MRI Conclusion: Sturge-Weber Syndrome.

Final Diagnosis: Sturge-Weber Syndrome (Proven).
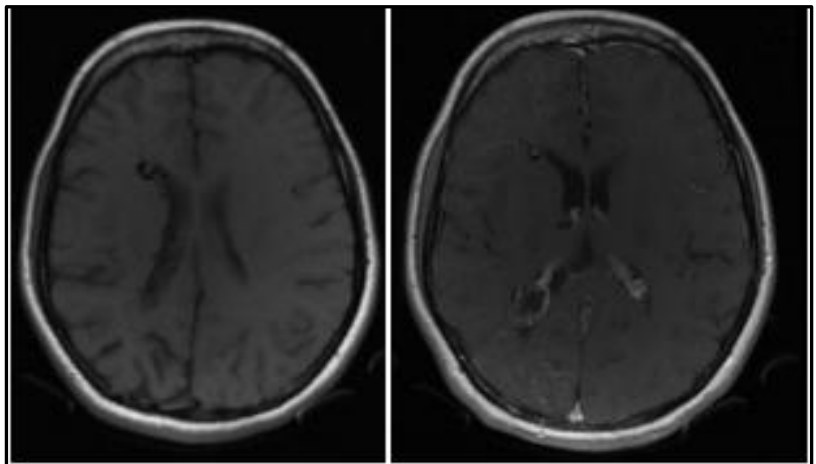

A

B

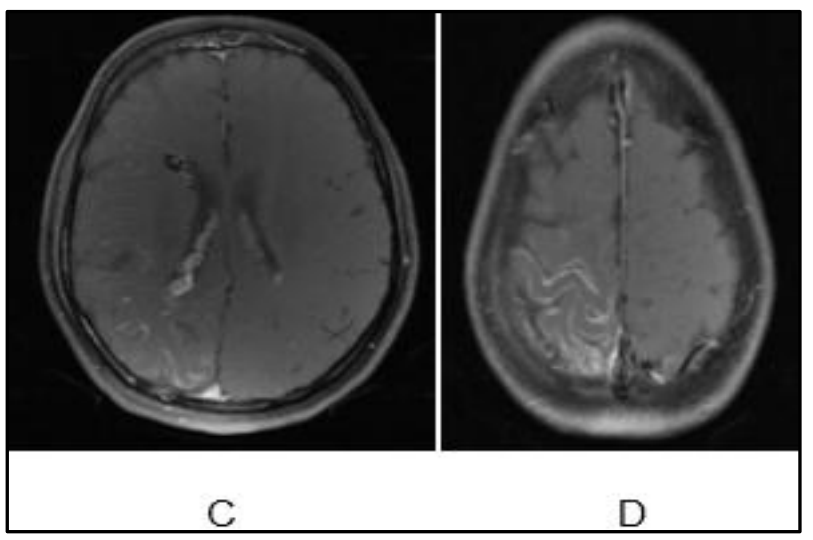

A. Axial: T1WI, B. and C and D. Post-Contrast T1WI Showing tram-track calcification with cerebral atrophy on right side with enlarged choroid plexus on right side. There is evidence of leptomeningeal enhancement.

\section{DISCUSSION}

Magnetic resonance imaging has become indispensable in the diagnostic work-up of epileptic patients, who present with 
recurrent seizures, which is amenable to both medical and surgical treatment.

About $15 \%$ - 30\% of patients with partial seizures are refractory to pharmacotherapy, which mandates the need to evaluate these patients with imaging studies to identify possible structural abnormalities, which may be responsible for seizures.

Identification of a structural substrate on magnetic resonance imaging guides further management, as the chance of being considered for surgical treatment is greatly enhanced when a structural abnormality is found on magnetic resonance imaging.

Approximately, $60 \%$ of patients become seizure free after surgery, the seizure free surgical outcome being $67 \%$ for mesial temporal sclerosis, $75 \%$ for neoplasms, $58 \%$ for cortical dysplasia.

With this background, we evaluated the magnetic resonance imaging features of patients presenting with seizures, either focal or generalised in paediatric age group between 0 - 16 yrs.

Our study included seventy three patients, who underwent magnetic resonance imaging. Out of the seventy three patients, the majority of patients belonged to 12 - 16 years' age group, the percentage being $32.88 \%$ in this age group. Rachana Chaurasia, Shuchi Singh, Sanchin Mathur ${ }^{17}$ have categorised the cause of epilepsy by the age of seizure onset in which majority are in this age group - similar to our study and the sensitivity of MR imaging in their study was given as $70.4 \%$.

About 45 (61.42\%) were males and 28 (38.57\%) were female patients. Our study included 43 patients with partial seizures and 30 patients with generalised seizures.

The epileptogenic focus/substrates which include infections, tumours, leucodystrophies, developmental malformations, gliosis, vascular malformations, encephalitis, infarct and thrombosis were identified based on the magnetic resonance imaging features for each of these lesions.

The MRI sensitivity is high in detecting lesions in partial seizure group, which is accounting to $59 \%$ in the study group when compared to generalised seizure group which is $41 \%$ and moreover the diagnostic yield of MRI was $72 \%$ in partial seizures which is only $40 \%$ in generalised seizure group.

Seizures as a consequence of infectious aetiology was seen in 13 (30\%) patients included in the study. About 8 (15\%) patients had evidence of neurocysticercosis, which appears as hypo-to-slightly hyperintense lesion on T1WI with ring enhancement and enhancing scolex on post-contrast images. These lesions usually follow CSF signal intensity on all sequences. Out of these, 8 patients had positive results for CSF IgM antibodies confirming NCC infection. Among them 5 $(10 \%)$ had tuberculoma, diagnosed on the basis of hypoisointense lesion on T1WI and hypointense to variable intensity lesion with surrounding oedema on T2WI, showing ring enhancement on post-contrast images. Routine chest radiograph was also performed for these patients, 3 of which showing positive signs of tuberculosis. CSF studies were performed showing raised protein in all 5 patients suggesting tuberculosis.

Rajasekhar $\mathrm{V}^{18}$ has reported increased frequency of neurocysticercosis than tuberculoma in our country in patients with seizures. In our study also, we found neurocysticercosis to be more common than tuberculoma.
Gliosis with or without encephalomalacia, Periventricular Leucomalacia (PVL), porencephaly and atrophy are varying responses of brain to any type of insult, mostly perinatal or in infancy but can occur later due to trauma, infection, infarct or haemorrhage. The result of insult during perinatal period depends upon the gestational age of patient, duration and severity of brain insult along with prenatal asphyxia, low birth weight, prematurity and toxaemia of pregnancy as predisposing factors. Those patients with perinatal insult usually presented with cerebral palsy, intractable seizures and developmental delay. In our present study, out of the 6 (13.9\%) cases, 2 cases showed encephalomalacia with gliosis, 2 cases had periventricular leucomalacia, 1 had hypoxic ischaemic encephalopathy and 1 patient had porencephaly. Periventricular leucomalacia, represents toxic injury to premyelinating oligodendrocytes because of cerebral ischaemia, reperfusion or both. Porencephalic cysts are congenital or acquired cavities within the cerebral hemisphere that usually - although not invariably - communicate directly with the ventricular system. They can be cortical or subcortical, unilateral or bilateral. The location often corresponds to territories supplied by the cerebral arteries.

Hui et al ${ }^{19}$ in their study of 100 patients with intractable epilepsy had found gliosis in $30 \%$ of patients and as the second most common cause of epilepsy. This is similar to our study.

Vascular diseases of the neonatal period include haemorrhage, venous thrombosis and arterial infarctions. Infarct contributed to $5(11.6 \%)$ cases in our study, of which 3 were arterial and 2 were venous in nature. In contrast to stroke in adults and older children, neonatal stroke often presents clinically with seizures but not with focal neurological deficiencies. Children can present stroke at any age. The incidence is higher under the age of 2 years and progressively decreases throughout adolescence. We did not find neonates/infants in the present series, probably due to difficulties in performing imaging exams during the acute phase of the disease at this time during the investigation. The common causes of strokes in children are rheumatic disease, infectious endocarditis, congenital heart disease, mitral valve prolapse, sickle cell disease, haemolytic uraemic syndrome and antiphospholipid antibody syndrome. In many cases, cause could not be determined. In our study, two children with infarct had Rheumatic Heart Disease and Congenital Heart Disease. In rest, cause could not be ascertained.

In our study among the 43 patients with positive magnetic resonance imaging, tumours were identified as the focal epileptogenic substrate in $4(9.3 \%)$ of the patients. Magnetic resonance imaging has nearly $100 \%$ sensitivity for identifying neoplastic lesions. In this study, there were 4 cases of tumours, 2 of them were of infratentorial origin - fourth ventricle masses causing dilatation of proximal ventricles showing variable/irregular contrast enhancement, one was dysembryoplastic neuroepithelial tumour and other was of hypothalamic hamartoma. Subsequent biopsy and surgery was performed, which confirmed the diagnosis as given on MR imaging.

Malformation of cortical development (pachygyria) with cortical dysplasia and open-lip schizencephaly, septo-optic dysplasia and heterotopias were found in $4(9.3 \%)$ patients of the study group in their first decade. Malformations of cerebral cortical development encompass a heterogeneous group of disorders frequently recognised on Magnetic Resonance 
Images (MRI). These types of disorders are a cause of human epilepsy.

We report three cases $(6.9 \%)$ of phakomatosis that was tuberous sclerosis in a 6-year-old male, who presented to Paediatric OPD with seizures, delayed mental development and skin manifestations. On MRI, scan showed subependymal nodules, cortical tubers and non-specific white matter changes. As many as $90 \%$ of patients with tuberous sclerosis have seizures, significant proportion of whom are refractory to medical therapy. One case was of a 13-year-old female who presented with seizures and port wine stain and on MRI revealed leptomeningeal enhancement with ipsilateral choroid plexus enlargement and tram track like calcifications along with cerebral hemiatrophy; and the other case was of neurofibromatosis-1 in a 14-year-old male who presented with seizures, headache and plexiform neurofibroma. On MRI imaging showed non-optic low-grade glioma, non-neoplastic hamartomas in bilateral basal ganglia and thalami.

Most of the epidemic encephalitis are caused by arboviruses and demonstrate regional and seasonal variation. Viral encephalitides are found all over the world with specific viruses being common in different geographical regions. In this series, we report three cases of encephalitis, one of which was Rasmussen's encephalitis which is a chronic inflammatory disease of unknown origin, whose aetiology and pathology was similar to viral encephalitis with MR imaging features corresponding to unilateral cerebral atrophy with T2 hyperintense areas in affected lobe along with restricted diffusion. The other two cases were of herpes simplex- 1 with typical MR imaging features of T2 hyperintensity of affected white matter and cortex showing variable enhancement and restricted diffusion (due to cytotoxic oedema), which was confirmed by PCR (polymerase chain reaction) DNA test, which reveals even tiny amounts of viral DNA being the diagnostic test for encephalitis.

Mesial temporal sclerosis was diagnosed based on the principal findings of volume loss and abnormal signal in the hippocampus with identification of loss of hippocampal architecture on inversion recovery sequence.

In the present study, hippocampal sclerosis was identified in $2(4.65 \%)$ patients. Similar results were shown by Lefkopoulos et al in their study of 120 patients with refractory seizures.

Minor findings such as atrophy of the ipsilateral fornix and mammillary body, ipsilateral dilatation of the temporal horns were also identified in our patients.

In the present study, white matter disease was seen in 2 (4.65\%) patients who presented with leucodystrophy. They are proven to be associated with epilepsy by Guissard et al and Hsieh et al, but their contribution has not been accounted for by any study. Since white matter does not have a generous blood supply as gray matter and is more susceptible to ischaemia (HIE, vasculitis secondary to infection), toxin and other insults. The most common location for the hyperintensities are the subcortical and periventricular white matter, optic radiations, basal ganglia and brain stem in decreasing order of frequency. The lesions are hyperintense on T2, proton density and FLAIR images and have well-defined but irregular margins. One case was proved to be Krabbe's leucodystrophy and the other as Wilson's disease.

Vascular malformations most commonly presenting with seizures include cavernous haemangioma and arteriovenous malformations. In our study, only one patient with cavernous haemangioma presenting with focal seizure was identified accounting for $2.3 \%$. Electroencephalography tells us that a seizure focus is present in the brain. It can also localise the focus as well as help to classify the seizures. However, it cannot characterise the underlying structural abnormality, which may be responsible for seizure in patients with partial epilepsy.

In present study electroencephalogram was done in all the cases, in which it was positive in 48 cases and normal in 25 cases. This was comparable to prior studies in 48 cases with positive EEG, 30 patients had abnormal MRI which was in accordance with previous studies, which showed that MRI abnormalities are usually associated with an abnormal EEG. It helps in establishing the diagnosis of seizures, characterising syndromes and providing prognosis. Out of 25 patients with normal EEG, 10 cases had abnormal MRI findings, so though the presence of an abnormal EEG in patient could suggest positive MRI finding, but obviously, a normal EEG doesn't rule out brain abnormality. This shows that MRI has higher chances of finding epileptogenic focus as compared to EEG alone, it goes in accordance with prior study by Kuzniecky and Knowlton ${ }^{20}$ 2002. It could be inferred that EEG could be helpful, but not alone.

The diagnostic yield of magnetic resonance imaging depends on many factors, one being the patient population it is applied to. The highest yield (65\% - 85\%) in detecting epileptogenic lesions is observed in patient groups with intractable partial epilepsy who are considered candidates for surgery.

In our study, the diagnostic yield of magnetic resonance imaging in the partial seizure group of paediatric age group between 0 - 16 yrs. is $59 \%$. Resta et al reported positive MRI in $51.3 \%$, Wang et al in $41.7 \%$ and Chang et al in $48.9 \%$. Our study shows a higher percentage, probably because of strict exclusion criteria, which shows that patient selection, plays an important role in MR positivity rates.

The most common lesion identified in children presenting with epilepsy being infection followed by gliosis. The localisation of epileptogenic focus can be made by clinical symptoms, electroencephalography, computed tomography, magnetic resonance imaging, functional magnetic resonance imaging, SPECT and PET studies. No single modality among these can definitively identify the seizure focus, especially those who are planned for epilepsy surgery and hence a combination of these should be used.

Computed tomography is widely available, very useful in the emergency setting and can detect major structural abnormalities and calcifications, but its sensitivity in the detection and characterisation of subtle cortical abnormalities, hippocampal lesions, leucodystrophies, vascular malformations, which is important in epilepsy imaging and also the detection of posterior fossa lesions is low.

On the other hand, magnetic resonance imaging with its superior soft tissue contrast, multiplanar imaging capability, lack of beam hardening artefacts is more sensitive in picking up subtle cortical abnormalities, hippocampal lesions as well as characterising lesions such as different types of tumours, infections with its advanced applications.

Functional magnetic resonance imaging, SPECT and PET also help to localise seizure focus. They are complementary to MRI. However, their availability restricts their routine usage, 
though they can be used in: 1) Patients with abnormal MRI with non-localising EEG or discordant EEG findings, 2) Multifocal magnetic resonance imaging (tuberous sclerosis), 3) Normal MRI with abnormal EEG.

From our study, we found that magnetic resonance imaging along with clinical history and electroencephalography should be used in the initial assessment of patients who are considered surgical candidates since the presence of focal pathology increases the chances of progression to successful surgical treatment.

\section{SUMMARY AND CONCLUSION}

The present study was performed in seventy three patients in the paediatric age group from 0 - 16 years who presented with clinical presentation of seizures either partial or generalised. They were imaged using an epilepsy protocol - magnetic resonance imaging. Magnetic resonance imaging should be considered in the initial evaluation of patients presenting with seizure, particularly intractable focal seizures because of its high sensitivity for epileptogenic substrates, superior soft tissue contrast, multiplanar capability, lack of beam hardening artefacts, lack of ionising radiation.

From our observations, we concluded that majority of children who presented with seizures belonged to age group from 11 - 16 yrs. Most of the patients were males. The proportion of patients presenting with partial seizures were higher than those with generalised seizures. The typical magnetic resonance signal intensity characteristics of epileptogenic substrates can be used to identify as well as diagnose the lesions specifically. Identification of a focal epileptogenic substrate is an important determinant in the selection of patients for epilepsy surgery, particularly in those cases in whom surgical resection is curative.

Abnormalities were identified in almost $60 \%$ of the study population, while $40 \%$ had normal magnetic resonance imaging.

Infectious aetiology including NCC and tuberculosis were common, in which NCC is the most commonly identified epileptogenic focus in children presenting with focal seizures. The second most common substrate was gliosis resulting from infarction, infection, trauma and hypoxia. This was followed by vascular diseases (infarct/thrombosis), congenital malformations, tumours, encephalitis, phakomatoses, mesial temporal sclerosis, leucodystrophy and cavernous angioma.

In the developmental malformations - cortical dysplasia, pachygyria, heterotopias, schizencephaly were identified as some of the causes for epilepsy. Only one patient in the study group had a vascular malformation, which was cavernous angioma.

So, in conclusion, our study shows that the diagnostic yield from magnetic resonance imaging is high in patients with refractory partial/focal seizures.

Thus, a combination of clinical information, electroencephalography and magnetic resonance imaging should be considered in the initial evaluation of patients presenting with refractory partial/focal seizures.

\section{REFERENCES}

[1] Gulati P, Jena A, Tripathi RP, et al. Magnetic resonance imaging in childhood epilepsy. Indian Pediatr 1991;28(7):761-5.
[2] The commission on classification and terminology of the ILAE. Proposal for revised clinical and electroencephalographic classification of epileptic seizures. From the commission on classification and terminology of the international league against epilepsy. Epilepsia 1981;22(4):489-501.

[3] The commission on classification and terminology of the ILAE. Proposal for revised classification of epilepsies and epileptic syndromes. Commission on classification and terminology of the international league against epilepsy. Epilepsia 1989;30(4):389-99.

[4] Sadler TW. Langmans medical embryology. $7^{\text {th }}$ edn. USA: Williams and Wilkins 1995:p 374.

[5] Vattipally VR, Bronen RA. Magnetic resonance imaging in epilepsy: strategies for successful interpretation. Neuroimaging Clin N Am 2004;14(3):349-72.

[6] Vattipally RV, Bronen RA. MR imaging of epilepsy: strategies for successful interpretation. Radiologic Clincs 2006;44(1):111-3.

[7] Sperner J, Schmauser I, Bittner R, et al. MR-imaging findings in children with sturge-weber syndrome. Neuropediatrics 1990;21(3):146-52.

[8] Raymond AA, Fish DR, Sisodiya SM, et al. Abnormalities of gyration, heterotopias, tuberous sclerosis, focal cortical dysplasia, microdysgenesis, dysembryoplastic neuroepithelial tumour and dysgenesis of the archicortex in epilepsy. Clinical, EEG and neuroimaging features in 100 adult patients. Brain 1995;118(Pt 3):62960.

[9] Huckman MS, Fox J, Topel J. The validity of criteria for the evaluation of cerebral atrophy by computed tomography. Radiology 1975;116(1):85-92.

[10] Montes JL. Lesionectomy of MRI detected lesions in children with epilepsy. Pubmed Pediatr Neuro Surg 1995;22(4):167-73.

[11] Van Passechen W, Sisodiyas S, Connelly A, et al. Quantitative hippocampal MRI and intractable temporal lobe epilepsy. Neurology 1995;45(12):2233-40.

[12] Salgado P, Brutto DOH, Talamás 0, et al. Intracranial tuberculoma: MR imaging. Neuroradiology 1989;31(4):299-302.

[13] Liu RS, Lemieux L, Bell GS, et al. Progressive neocortical damage in epilepsy. Ann Neurol 2003;53(3):312-24.

[14] Tang P. Annals academy of medicine Singapore 2008;37:397-401.

[15] Brodtkorb E, Nilsen G, Smevik O, et al. Epilepsy and anomalies of neuronal migration: MRI and clinical aspects. Acta Neurol Scand 1992;86(1):24-32.

[16] Lehericy S, Dormont D, Semah F, et al. Developmental abnormalities of the medial temporal lobe in patients with temporal lobe epilepsy. Am J Neuroradiol 1995;16(4):617-26.

[17] Chaurasia R, Singh S, Mathur S, et al. Imaging in pediatric epilepsy: spectrum of abnormalities detected on MRI JEMDS 2013;2(19):3377-87.

[18] Rajasekhar V. Solitary cerebral cysticercus granuloma. Epilepsy 2003;44(1):25-8.

[19] Hui AC, Lam JM, Chan YL, et al. Role of magnetic resonance imaging for preoperative evaluation of patients with refractory epilepsy. Hong Kong med J 2003;9(1):20-4.

[20] Kuzniecky RI, Knowlton RC. Neuroimaging of epilepsy. Semin Neurol 2002;22(3):279-88. 